$\underline{\text { Original Articles }}$

\title{
Assortment of adrenocortical tumours
}

\author{
R Ajanthan ${ }^{1}$, Sanath P Lamabadusuriya ${ }^{2}$ \\ Sri Lanka Journal of Child Health, 2002; 31: 9-13
}

(Key words: adrenocortical tumour, virilisation, hypertension, Cushing syndrome)

\begin{abstract}
Six cases of adrenocortical tumours seen in children in a space of 6 years are presented. All 6 children had hypertension at presentation in addition to virilisation, Cushing syndrome or both. Mean age at presentation was 5 years. Sex ratio was equal. Duration of symptoms varied from 2 months to seven years. Diagnosis in all six cases was made on ultrasound examination of the adrenal bed. Surgical resection was done in all 6 children and 5 were referred for chemotherapy. Mean weight of the tumours was $138 \mathrm{~g}$. Two of them were alive until they were lost for follow up. One was followed up for thirty months.
\end{abstract}

\section{Introduction}

Adrenal tumours are rare in childhood ${ }^{1}$. Although nonsecreting tumours have been described, the majority of paediatric patients have presented with signs of hormonal activity. In this paper, diagnosis and management of the six cases are summarized.

\section{Method}

At presentation, all cases were carefully assessed with a detailed history, examination and basic investigations to arrive at a diagnosis. Height-for-age and weight-for-age were assessed from standard growth charts and boneage from hand and wrist radiographs by the Greulich and Pyle method ${ }^{2}$. Serum electrolytes, ultrasound scan of the abdomen and intravenous urograms were done to diagnose and assess the lesion. Occasionally computerized tomograms were requested too.

Urinary excretion of ketosteroids were done but more specific tests like serum levels of testosterone, dehydroepiandrosterone or adrenocortical suppression tests were not performed due to financial constraints and non-availability of facilities.

\section{Case 1}

A 10 year old girl was referred for management of

${ }^{1}$ Senior Lecturer, ${ }^{2}$ Senior Professor, Department of
Paediatrics, Faculty of Medicine, University of
Colombo.

hypertension. She had a history of increasing pubic hair and clitoromegaly since 3 years of age and facial hair with a deep voice of 2 years duration. She had a masculine build with hirsutism. Her height-age and weight-age were 9 years whilst her bone-age was 13 years. She was hypertensive with a blood pressure of 200/120 $\mathrm{mm} \mathrm{Hg}$ and examination of her external genitalia revealed pubic hair of Tanner stage 4 and clitoromegaly of $5.0 \mathrm{~cm}$. Her breasts were prepubertal and no masses were felt in the abdomen. Hypokalaemia and elevated levels of ketosteroids in the urine confirmed adrenal involvement and the advanced bone age was supportive. Ultrasound scan visualised a right adrenal mass of $3.7 \mathrm{~cm} \times 3.5 \mathrm{~cm}$.

Laparotomy was done with perioperative steroid coverage and an encapsulated tumour weighing $140 \mathrm{~g}$ was removed. Histology revealed capsular invasion with several atypical features.

During follow up she again developed hypertension and on further investigation was noted to have hypercalcaemia with bilateral nephrocalcinosis. She was last reviewed $21 / 2$ years after her initial presentation and was found to be hypertensive with normal renal function. We were unable to do PTH and PTH related polypeptide, which may have established the cause of her nephrocalcinosis.

\section{Case 2}

An 18 month old boy presented to us with clinical evidence of hypercortisolism of 2 months duration. He was obese, had a moon face and cutaneous striae but had no evidence of sexual precocity. His height-age was 2 years, weight-age 5 years and bone-age 1 year. He was hypertensive. There was no history of steroid ingestion and a hormone secreting tumour was suspected. Ultrasound examination revealed a right suprarenal mass.

At laparotomy under perioperative steroid cover, a disc shaped tumour of $4.0 \mathrm{~cm} \times 6.0 \mathrm{~cm} \times 0.5 \mathrm{~cm}$ weighing $100 \mathrm{~g}$ was removed. It was found to be adherent to the caudate lobe of the liver. Histology revealed no evidence of malignancy and he was discharged on antihypertensive drugs which were tailed off over the next three to four weeks. 
4 months later, he was readmitted with a history of rapid weight gain and focal convulsions. On examination, he had pubic hair of Tanner stage 2 with a blood pressure of 160/130 mm Hg. An urgent scan of the abdomen revealed a recurrence of tumour in the right adrenal bed. His condition deteriorated over the next two weeks and he died. A limited post mortem examination revealed findings similar to the scan. (Figure 1)

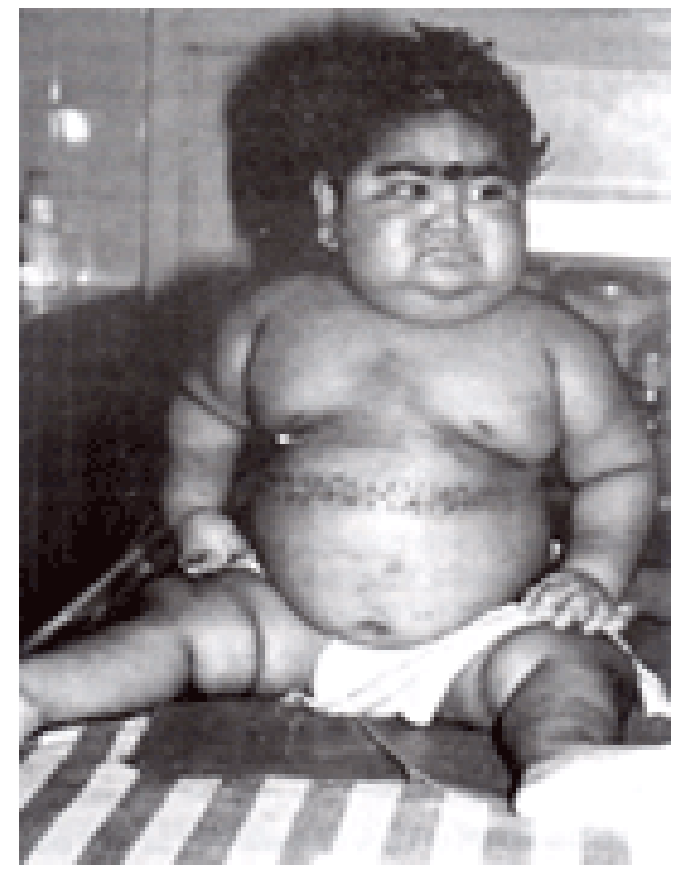

Figure 1 Case 2 with evidence of hypercortisolism

\section{Case 3}

A 4 year old boy was referred by a dermatologist for increasing acne over the face and upper chest of 1 year duration associated with a deep voice. On examination, he had features of masculinisation and extensive acne. He had a height-age of 5 years, weight-age of 8 years and bone-age of 12 years. He was hypertensive. Examination of the genitalia showed a large phallus with pubic hair of Tanner stage 2 with prepubertal sized testes. No masses were felt in the abdomen. (Figure 2)

Investigation revealed hypokalaemia and eleva-ted urinary ketosteroids. An abdominal x-ray showed calcification over the right adrenal area. Ultrasound examination confirmed a mass in the right adrenal bed. At laparotomy, an encapsulated tumour weighing 190g was removed and recovery was uneventful. Histology showed capsular invasion with atypical features.

He was referred to Cancer Institute, Maharagama for chemotherapy which was given for over three months. He died of pulmonary secondaries one year after the initial presentation.

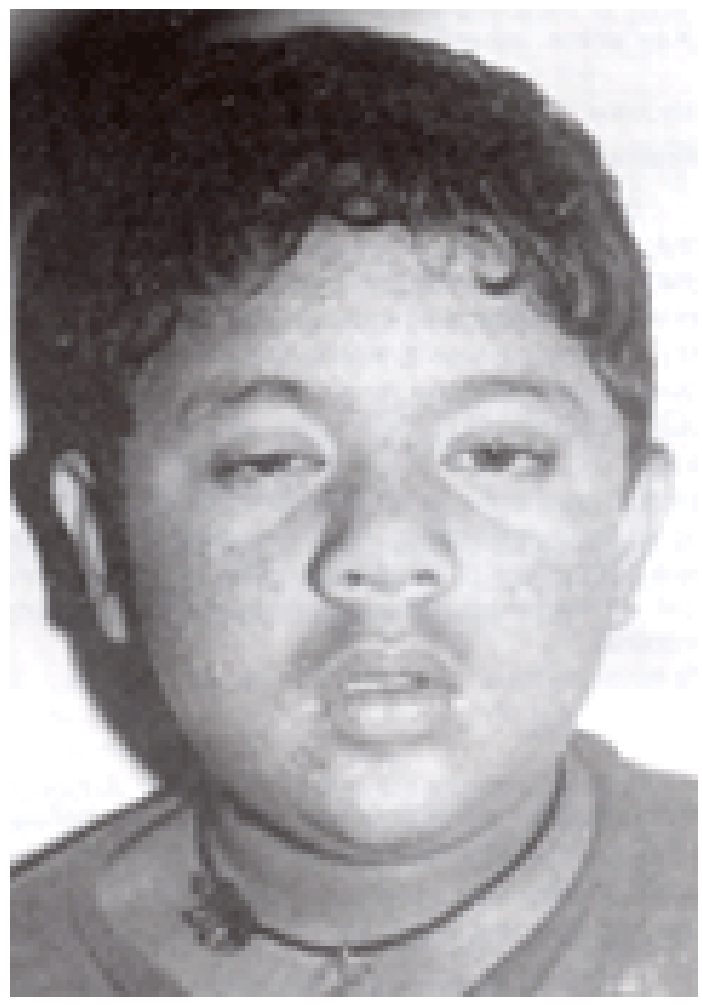

Figure 2. Case 3 with evidence of androgen excess

\section{Case 4}

A 5 year old girl was transferred to us with complications of hypertensive encephalopathy. Mother had noticed rapid increase in weight, appearance of pubic hair and changes in the external genitalia over the past six months. Two weeks prior to transfer she developed features of hypertensive encephalopathy and remained unconscious. There was no history of increased steroid intake.

On examination, she had marked Cushingoid features with a height-age of 4.5 years, weight-age of 7 years and bone-age of 3 years. She was on antihypertensive drugs but had cortical blindness. Pubic hair was of Tanner stage 2 with evidence of clitoromegaly. Examination of the abdomen revealed a vague mass below the left hypochondrium. Ultrasound of the abdomen and computed tomographic scan of abdomen and brain showed a tumour in the left adrenal bed and infarcts in the occipital cortex.

At laparotomy, a well encapsulated tumour weighing $175 \mathrm{~g}$ was removed from the left adrenal bed. Perioperative steroids were given. Histology was suggestive of adrenal carcinoma with capsular invasion and she was transferred to the Cancer Institute, Maharagama for further management. 


\section{Case 5}

A 9 month old girl was transferred to us with increased hair growth of the face, back and genitalia of 4 months duration. She was the second child of a nonconsanguineous marriage with no history of previous hospital admissions or drug ingestion. Her height-age and weight-age were 5 months, and bone-age was 9 months. She was hirsute with Cushingoid facies and acne but no axillary hair or breast development. She was hypertensive with a palpable lump $5 \mathrm{~cm}$ below the right costal margin.

Ultrasound examination revealed a right adrenal tumour and an intravenous urogram showed normal renal function. At laparotomy, a $7 \mathrm{~cm} \times 4 \mathrm{~cm} \times 5 \mathrm{~cm}$ well encapsulated mass weighing $108 \mathrm{~g}$ was removed. Right kidney, IVC and liver were normal. She was managed with perioperative steroids and discharged on day 7 after surgery. Histology revealed adrenocortical carcinoma and she was referred for chemotherapy and died while receiving treatment at Cancer Institute, Maharagama.

\section{Case 6}

A 9 year old boy was referred for further management of Cushing syndrome. He had presented with congestive cardiac failure secondary to hypertension. On admission, he was comfortable but obviously Cushingoid. His height-age was 5 years, weight-age was 5.5 years and bone-age was 4 years.

His hypertension was controlled with nifedipine and hydralazine. Initially hypokalaemia was evident but ketosteroid excretion was only minimally increased.

Ultrasound examination showed a left adrenal mass and at laparotomy with perioperative steroid cover a wellencapsulated tumour $8 \mathrm{~cm} \times 7 \mathrm{~cm}$ x $6 \mathrm{~cm}$ weighing $120 \mathrm{~g}$ was removed. Histology showed vascular invasion with a mitotic count of $4 / 50 \mathrm{hpf}$. He was referred to the Cancer Institute Maharagama for further management where he died of hypertensive encephalopathy.

Clinical features are summarised in Table 1.

Table1

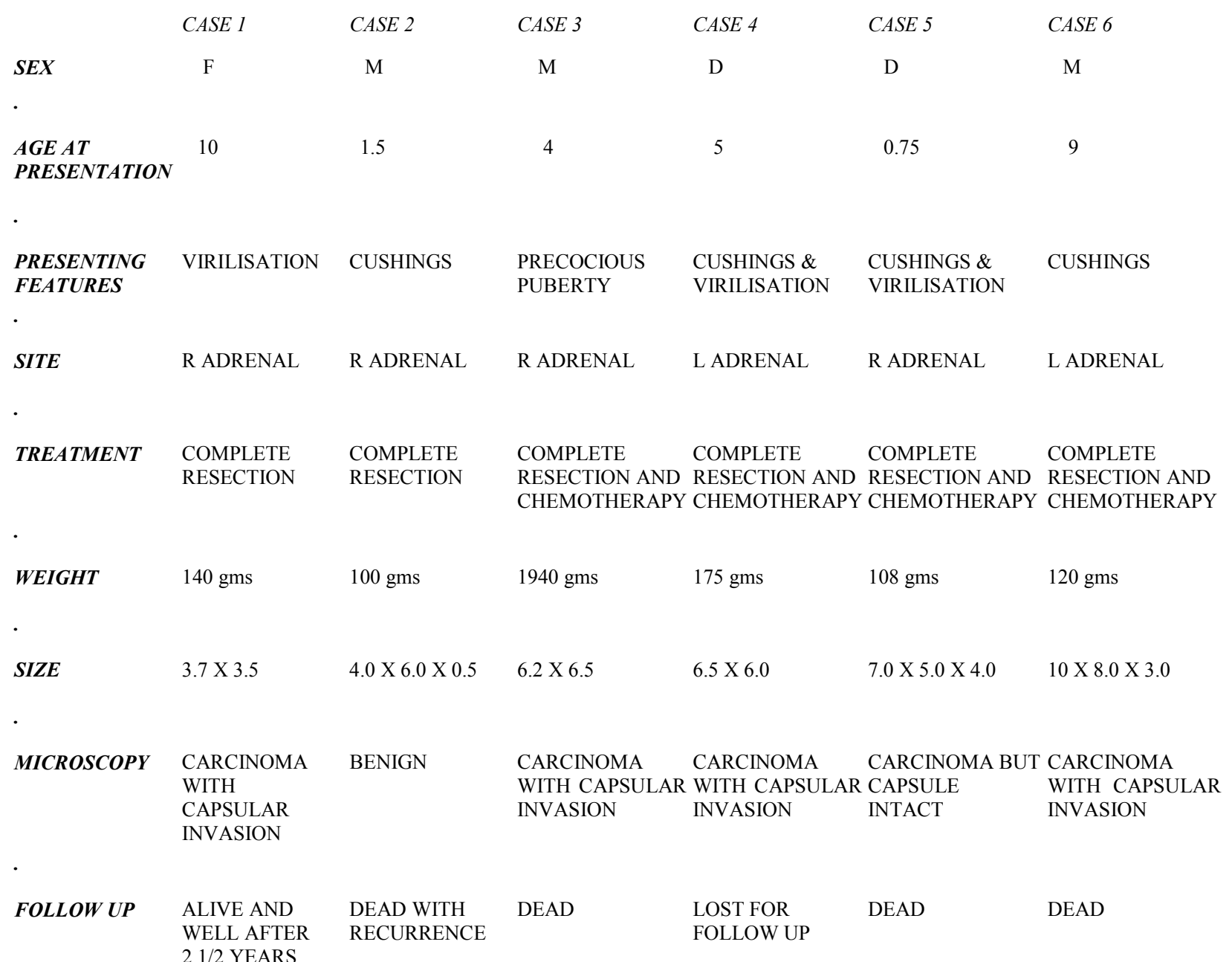




\section{Discussion}

The first case of adrenal tumour was documented in 1865. "General melasma and short hair over the entire body of a 3 year old child with conversion of the left supra renal capsule into a large malignant tumour; the external organs of generation resembling that of adult life". was the original description ${ }^{3}$.

The adrenal cortex develops from the mesoderm and a whole gland weighs only $3-5 \mathrm{~g}$. Anatomical size is grossly incomparable to its physiological function without which life is incompatible. Tumours of the adrenal cortex are mostly unilateral, carcinoma being three times greater than adenoma. It is commoner in girls and majority present below 5 years of age.

Tumours, in general, cause a catabolic effect with loss of body mass and weight. But adrenocortical tumours are an exception to this rule as they secrete increased amounts of androgens and/or other steroids causing an anabolic effect. About $66 \%$ of cases have presented with virilisation and the balance with hypercortisolism. These tumours are rare in childhood and in some instances non-secretory tumours have been reported. In our series all cases presented with either virilization, Cushing syndrome or both.

Adrenocortical tumours have been found to be associated with hemihypertrophy ${ }^{4}$, astrocytoma ${ }^{5}$, cutaeneous lesions, neurofibromatosis ${ }^{6}$ and Beckwith-Wiedemann $\quad$ syndrome ${ }^{7}$ Sibship relationship has been noted with these tumours ${ }^{8}$ and they have been associated with congenital adrenal hyperplasia too 9 . We did not find any association with the above in our series.

Recently, various genetic predispositions have been found to be associated with adrenocortical carcinoma. These are

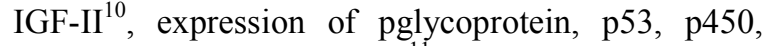
EGF-1, K1-67 and PCNA ${ }^{11}$. Immunocytochemical differences have been developed with the use of monoclonal antibody $\mathrm{D} 11^{12}$ and $\mathrm{MHC}$ class II expression has been used to assess dignity of these tumours $^{13}$. Non availability of these test kits have prevented us from conducting further investigation. Magnetic resonance imaging has been found to be more useful in demarcating the tumour ${ }^{14}$.

Most of these cases present with virilisation with or without hypercortisolism and much less frequently with feminization, gynaecomastia or hyperaldosteronism. In our series all cases presented with either virilization, hypercortisolism or both. Significantly, all six patients had hypertension on presentation and it is worth postulating whether the association of hypertension with virilism is usually due to an underlying adrenocortical tumour rather than hyperplasia.

Androgen excess leading to precocious puberty in the male is adrenal in origin when the testes are small and testicular in origin when they are large. Androgen excess in girls leads to a virilized female. Hypercortisolism in childhood is mostly due to iatrogenic steroid ingestion and if this is excluded, then adrenal tumours are the next commonest. Typical features are growth arrest, moon facies, obesity with cutaneous striae and other skin changes.

In our series diagnosis was established by simple non-invasive investigations like $\mathrm{x}$ - ray and ultrasound scan of the abdomen. Presently MRI scans are done for a more definitive diagnosis. Non availability of other hormonal investigations was not a constraint to our pre-operative diagnosis.

Treatment consisted of removal of the tumour en masse in every instance. Some were referred for chemotherapy and further evaluation. But it has been shown that place of radiotherapy or chemotherapy has been doubtful in these cases ${ }^{15}$. Histology of these tumours played a very minor role in deciding prognosis (e.g. case 2), compared to the weight of the tumour, which plays a significant role in deciding prognosis $^{16}$. In our series the prognosis has been poor with four out the six dead within two years of diagnosis.

\section{Acknowledgements}

The authors wish to acknowledge late Dr. Gamini Karunaratne, Dr. Y. Kulasekara, Dr. Ranjan Dias Consultant Paediatric Surgeons for performing surgery, Dr. I. N. A. Gooneratne Consultant Radiologist for the radiological studies and Dr. Senarath Jayathileke Consultant Oncologist for chemotherapy.

\section{References}

1. Javadpour N, Woltering E A, Brennan M F. Adrenal neoplasms. Current problems in Surgery, 1980; 17: 1.

2. Greulich W W, Pyle S E. Radiologic atlas of skeletal development of the hand and wrist, ed 2, Stanford Ca, Stanford University Press, 1959. 
3. General melasma and short hair over the entire body of a child of three years, with conversion of the left supra renal capsule into a large malignant tumour; the external organs of generation resembling that of adult life. Lancet 1865; 1: 175.

4. Haicken B N, Schulman N H, Schneider K M. Adrenocortical carcinoma and congenital hemihypertrophy. Journal of Paediatrics 1973; 83: 284.

5. Fraumeni J M, Miller R W. Adrenocortical neoplasms with hemihypertrophy, brain tumours and other disorders. Journal of Paediatrics 1967; 70: 129.

6. Gershanik J J, Elmore M, Levkoff A $\mathrm{H}$. Congenital concurrence of adrenal cortical tumour, ganglioneuroma, and toxoplasmosis. Paediatrics 1951; 51: 705.

7. Wiedemann H R. Tumours and hemihypertrophy associated with Wiedemann - Beckwith syndrome. European Journal of Paediatrics 1983; 141: 129.

8. Steiner M M. Clinical approach to endocrine problems in children. St Louis, CV Mosby Co, 1970, p282.

9. Pang S, Becker D, Cotelingam $J$, et al. Adrenocortical tumour in a patient with congenital adrenal hyperplasia due to 21hydroxylase deficiency. Paediatrics 1981; 68: 242 .

10. Schofield P N, Nystrom A, Smith J, et al: Expression of a high molecular weight form of insulin _ like growth factor II in a Beckwith Wiedemann syndrome associated adrenocortical adenoma. Cancer 1995; 94(1):71-7.
11. Haak H R, et al. Expression of P-glycoprotein in relation to clinical manifestation, treatment and prognosis of adrenocortical cancer. European Journal of Cancer 1993; 29A(7): 1036-8.

12. Schroder S, et al. Immunocytochemical differential diagnosis of adrenocortical neoplasms using the monoclonal antibody D11. Virchows Archives of Anatomical Pathology and Anatomical Histopathology 1990; 417(2): 89-96.

13. Marx C, et al. MHC Class II expression - a new tool to assess dignity in adrenocortical tumours. Journal of Clinical Endocrinology and Metabolism 1996; 81(12): 4488-91.

14. Hanson J A, et al. Magnetic resonance imaging of adrenocortical adenomas in childhood: correlation with computed tomography and ultrasound. Paediatric Radiology 1996; 26(11): 794-9.

15. Arico M, Bossi G, Livieri C, et al. Partial response after intensive chemotherapy for adrenal cortical carcinoma in a child. Med Paediatric Oncology 1992; 20(3): 246-8.

16. Wolthers O D, Cameron F J, Scheimberg I, et al: Androgen secreting adrenocortical tumours. Archives of disease in childhood 1999; 80(1): 46-50. 
